\title{
A NOTE ON FINITE GROUPS HAVING A FIXED-POINT-FREE AUTOMORPHISM
}

\author{
MARTIN R. PETTET
}

ABSTRACT. A fusion result of Glauberman has as a consequence the fact that a finite group admitting a fixed-point-free automorphism has a normal Sylow 2-subgroup (and in particular, is solvable) if all nontrivial fixed-point subgroups have odd order.

The aim of this note is to point out a simple consequence of a recent fusion result of Glauberman which has an application to the problem of finite groups admitting a fixed-point-free automorphism. The general conjecture is that such groups are solvable. (See, for example, [3] and [2, Chapter 10].) We prove the following

Theorem. Let $G$ be a finite group admitting a fixed-point-free automorphism $\sigma$ such that the fixed-point subgroup of each nontrivial power of $\sigma$ has odd order. Then $G$ is 2-closed.

Note that 2-closure implies solvability by the Feit-Thompson theorem.

The argument is essentially a generalization of one used in the proof of Theorem 4.1 of [3].

Let $Q d(p)$ be the semidirect product of the 2-dimensional vector space over $G F(p)$ with its special linear group, and write $F(p)$ for the normalizer in $Q d(p)$ of a Sylow $p$-subgroup. Corollary 1 of Glauberman's paper [1] then states that if $p$ is an odd prime, $P$ is a Sylow $p$-subgroup of $G$, and $F(p)$ is not involved in $N_{G}(Z(J(P)))$, then $Z(J(P))$ controls strong fusion in $P$ with respect to $G$.

Lemma. A finite group $G$ is 2-closed if and only if $N_{G}(Z(J(P)))$ is 2-closed for every odd order Sylow subgroup $P$ of $G$.

Proof. The "only if" direction is trivial, so assume that $N_{G}(Z(J(P)))$ is 2-closed for every odd order Sylow subgroup $P$. A Frattini argument shows that for odd primes $p$, normalizers of $p$-subgroups are preserved under the natural map from $G$ to $G / O_{2}(G)$, so induction allows us to assume $O_{2}(G)$ $=1$. By Baer's theorem [2, Theorem 3.8.2], if $x$ is an involution in $G$, the dihedral group $\left\langle x, x^{g}\right\rangle$ is not a 2 -group for some element $g \in G$, so con-

Received by the editors June $17,1974$.

AMS (MOS) subject classifications (1970). Primary 20D10; Secondary 20D45.

Key words and phrases. Fixed-point-free automorphism, 2-closed, $Z(J(P))$ controls, strong fusion in $P$. 
jugation by $x$ must invert a nontrivial $p$-element $y$ for some odd prime $p$. But if $P$ is a Sylow $p$-subgroup of $G$ containing $y$, the hypothesis that $N_{G}(Z(J(P)))$ is 2-closed implies both that $F(p)$ is not involved in $N_{G}(Z(J(P)))$ and also that no element of $N_{G}(Z(J(P)))$ can invert $y$. These two conclusions are in contradiction with the result of Glauberman mentioned above.

Before proving the theorem, we recall that if a finite group $G$ admits a fixed-point-free automorphism $\sigma$, then for each prime $p, G$ contains a unique $\sigma$-invariant Sylow $p$-subgroup which in turn contains every $\sigma$-invariant $p$-subgroup of $G$.

Proof of Theorem. We argue by induction on the order of $G$. If $N_{G}(Z(J(P))) \neq G$ for every odd order $\sigma$-invariant Sylow subgroup $P$ of $G$, we may simply apply induction and the above Lemma. Otherwise, $O_{p}(G) \neq 1$ for some odd prime $p$ so, if $S$ is the $\sigma$-invariant Sylow 2-subgroup of $G$, induction applied to $G / O_{p}(G)$ yields that $S O_{p}(G) \triangleleft G$. But by hypothesis, the action of $\sigma$ on $S O_{p}(G)$ is such that the fixed points of every nontrivial power of $\sigma$ lie in $O_{p}(G)$, so [4, Lemma 1] implies that $S O_{p}(G)=S \times O_{p}(G)$. Thus, $S \triangleleft G$ as required.

\section{REFERENCES}

1. G. Glauberman, A sufficient condition for p-stability, Proc. London Math. Soc. (3) 25 (1972), 253-287. MR 47 \# 1938. \#229.

2. D. Gorenstein, Finite groups, Harper and Row, New York, 1968. MR 38

3. E. W. Ralston, Solvability of finite groups admitting fixed-point-free automorphisms of order rs, J. Algebra 23 (1972), 164-180. MR 46 \#1902.

4. B. Scimemi, Finite groups admitting a fixed-point-free automorphism, J. Algebra 10 (1968), 125-133. MR 37 \#5289.

DEPARTMENT OF MATHEMATICS, UNIVERSITY OF WISCONSIN, MADISON, WISCONSIN 53706 